\title{
Clinical Characteristics of Patients on Long-term Hemodialysis
}

\author{
Amna Hamid, Murtaza F. Dhrolia, Ruqayya Qureshi, Salman Imtiaz and Aasim Ahmad
}

\begin{abstract}
Objective: To study the clinical characteristics of patients on hemodialysis for more than ten years in order to highlight the modifiable factors that may improve survival in low socioeconomic status.

Study Design: Observational cross-sectional study.

Place and Duration of Study: Department of Nephrology, The Kidney Centre Postgraduate Training Institute (TKCPGTI), Karachi, from November 2017 to January 2018.

Methodology: Clinical records of 38 patients, who have been on thrice weekly hemodialysis for more than ten years at the study centre with registration period from 1989 to 2018, were reviewed. The participants were placed in three groups according to duration of hemodialysis: 10 to 15 years; 15 to 20 years, and $>20$ years. Background information (age, gender, age at initiation of hemodialysis, cause of renal failure), dialysis dose (urea reduction ratio), anemia (hemoglobin), bone-mineral metabolism (calcium, phosphate and iPTH), and nutrition (albumin) were evaluated. The parameters were compared among the groups and with respect to K/DOQI and EBPG guidelines.

Results: Mean age at institution of hemodialysis was 33.5 years. The most common cause of End Stage Renal Disease (ESRD) was of unknown etiology (bilateral small shrunken kidneys). Chronic sclerosing glomerulonephritis was the second most common cause. The mean hemoglobin level was $10.98 \pm 1.4 \mathrm{~g} / \mathrm{dL}$. Mean phosphate, calcium and iPTH were $4.75 \pm 1.34 \mathrm{mg} / \mathrm{dL}, 9.62 \pm 0.64 \mathrm{mg} / \mathrm{dL}$ and $281.5 \mathrm{pg} / \mathrm{ml}$, respectively. Serum albumin was $3.66 \pm 0.39 \mathrm{mg} / \mathrm{dL}$. These indexes were comparable to recommended guidelines.

Conclusion: Clinical characteristics and other profiles of patients on hemodialysis for more than 10 years are comparable to the standard K/DOQI and EPBG guidelines. We believe that complying the standard guidelines for management of patients on chronic hemodialysis can result in better long-term outcome and survival in our population.
\end{abstract}

Key Words: Clinical characteristics, Long-term hemodialysis, Modifiable factors, Improve survival.

\section{INTRODUCTION}

Developing countries including Pakistan face a silent epidemic of end stage renal disease (ESRD) in current globally growing burden of chronic kidney disease. 1 Pakistan lacks a national renal registry to provide a reliable estimate of ESRD prevalence. However, a report estimated 150 patients with ESRD per annum per million population; which accounts for 16000 new cases each year. ${ }^{2}$

There are limited centres that provide renal transplantation to disproportionately growing ESRD population. Hence, hemodialysis remains a promising treatment modality. Global data demonstrates that survival on long term hemodialysis is generally poor. ${ }^{3}$ Hence, sophisticated management of ESRD to reduce morbidity and mortality on long term hemodialysis is the greatest challenge for nephrologists of this era.

Department of Nephrology, The Kidney Centre Postgraduate Training Institute (TKC-PGTI), Karachi, Pakistan

Correspondence: Dr. Amna Hamid, Department of Nephrology, The Kidney Centre Postgraduate Training Institute (TKC-PGTI), 197/9, Rafiqui Shaheed Road, Karachi 75530, Pakistan

E-mail: amnahamid.88@gmail.com

Received: July 27, 2018; Accepted: October 30, 2018
In recent years, several guidelines, particularly, Kidney Disease Outcomes Quality Initiative (K/DOQI) and European Best Practices Guidelines for Hemodialysis (EBPG), 4,5 have been devised to improve quality of healthcare and long-term outcomes on hemodialysis. While evidences suggest that advanced dialysis techniques and therapeutic strategies have resulted in increased survival on hemodialysis worldwide, $3-5$ Pakistan still strives to provide quality dialysis care to majority of patients and survival rate on hemodialysis remains poor. 1,2

Due to limited resources, majority of healthcare facilities in Pakistan fail to provide adequate dialysis and patient management, according to recommended guidelines. ${ }^{1,2}$ This could explain poor survival on hemodialysis in this region. Local data on chronic hemodialysis is scarce. Furthermore, long term survival and factors determining the outcome of chronic hemodialysis are also not well documented.

Therefore, the aim of present study was to observe the basic characteristics of patients on maintenance hemodialysis for more than ten years in order to ascertain modifiable factors in management of ESRD patients that may improve survival on chronic hemodialysis.

\section{METHODOLOGY}

This was an observational study carried out in the Department of Nephrology, The Kidney Centre Post- 
graduate Training Institute (TKC-PGTI). The ethical approval was sought from the Ethical Review Committee to review medical records of 38 patients on maintenance hemodialysis. All patients underwent a four-hour session of thrice per week hemodialysis for more than ten years at The Kidney Centre Postgraduate Training Institute during the years 1989 to 2018 . The study subjects were divided into three categories: 10 to 15 years, 15 to 20 , and $>20$ years, based on the duration on hemodialysis. Patients who had undergone renal transplantation or peritoneal dialysis at any point were excluded.

Background information including age, gender, age at initiation of hemodialysis, cause of renal failure were obtained from medical records at the study centre. Urea reduction ratio was taken as index of hemodialysis adequacy. Bone mineral metabolism (serum phosphate, calcium, iPTH), anemia management (serum hemoglobin and ESA dose), and nutrition (serum albumin and reduced interdialytic weight gain) were also assessed.

Background information and other parameters were compared among the three groups in order to determine effect of duration of hemodialysis on these factors in view of recent KDOQI recommendations.

Data analyses were performed by using software IBM license SPSS version 21. Shapiro-Wilk test was applied to determine the normality of data. Mean and standard deviation (SD) were reported for normally distributed continuous variables; while for skewed data, medians with interquartile range (IQR) were described. Number with percentage was reported for categorical parameters. To detect any difference among groups of duration of hemodialysis, one-way ANOVA was applied for normally distributed variables. Kruskal-Wallis test was used for skewed parameters. Level of significance was considered at $<0.05$.

\section{RESULTS}

A total of 38 patients, who were on hemodialysis for minimum of ten years, were enrolled in the present study. Most patients were females $(n=26,68.4 \%)$, whereas $12(31.6 \%)$ were males. The mean age of the patients at the time of enrolment was 47 years and the mean age of initiation of hemodialysis was 33.5 years. It was noted that the age at onset of dialysis was significantly younger in patients who were on hemodialysis for longer duration $(p=0.039)$.

The predominant cause of ESRD in this study was unknown etiology; however, glomerulonephritis and polycystic kidney disease were seen as prominent etiologic factors of the disease. It was observed that none of the patients, who survived hemodialysis therapy for more than 15 years, had diabetic nephropathy. Table I compares the background information among the three groups on long term hemodialysis.
Table I: Background information.

\begin{tabular}{|c|c|c|c|}
\hline & \multicolumn{3}{|c|}{ Duration of hemodialysis (years) } \\
\hline & $10-<15$ & $15-20$ & $>20$ \\
\hline Number n (\%) & $20(52.6)$ & $11(28.9)$ & $7(18.4)$ \\
\hline Age (years) & $50.7 \pm 13.7$ & $48 \pm 13.9$ & $49 \pm 11.1$ \\
\hline Age at initiation of hemodialysis & $38.65 \pm 13.25^{*}$ & $29.6 \pm 13.9^{*}$ & $25 \pm 9.58^{*}$ \\
\hline \multicolumn{4}{|l|}{ Gender n (\%) } \\
\hline Male & $5(25)$ & $4(36.4)$ & $3(42.9)$ \\
\hline Female & $15(75)$ & $7(63.6)$ & $4(57.1)$ \\
\hline \multicolumn{4}{|l|}{ Cause of ESRD n (\%) } \\
\hline Unknown & $8(40)$ & $6(54.5)$ & $4(57.1)$ \\
\hline Glomerulonephritis & $2(10)$ & $3(27.3)$ & $1(14.3)$ \\
\hline Polycystic kidney disease & $3(15)$ & $2(18.2)$ & 0 \\
\hline Diabetes mellitus & $2(10)$ & 0 & 0 \\
\hline Hypertension & $2(10)$ & 0 & 0 \\
\hline Renal stone disease & $2(10)$ & 0 & 0 \\
\hline Interstitial nephritis & 0 & 0 & $1(14.3)$ \\
\hline Other & $1(5)$ & 0 & $1(14.3)$ \\
\hline \multicolumn{4}{|l|}{ Comorbidity n (\%) } \\
\hline Diabetes mellitus & $4(20)$ & 0 & 0 \\
\hline Hypertension & $16(80)$ & $10(90)$ & $5(71.4)$ \\
\hline Ischemic heart disease & $1(5)$ & $1(9)$ & $2(28.5)$ \\
\hline Cerebrovascular disease & $1(5)$ & 0 & $1(14.3)$ \\
\hline Chronic liver disease & $2(10)$ & $2(18.2)$ & $3(42.8)$ \\
\hline Thyroid disorder & $2(10)$ & $2(18.2)$ & 0 \\
\hline
\end{tabular}

${ }^{*} P=0.039 ; \quad E S R D$ : end stage renal disease.

Table II: Continuous demographic and clinical parameters of HD patients.

\begin{tabular}{lc}
\hline Variables & Mean \pm SD/ Median, IQR \\
\hline Age of patients (years) & $47,22.5$ \\
Age at initiation of HD (years) & $33.5 \pm 13.7$ \\
URR $(\%)$ & $73,10.95$ \\
$\mathrm{Hb}(\mathrm{g} / \mathrm{dL})$ & $10.98 \pm 1.4$ \\
Calcium $(\mathrm{mg} / \mathrm{dL})$ & $9.62 \pm 0.64$ \\
Phosphorus $(\mathrm{mg} / \mathrm{dL})$ & $4.75 \pm 1.34$ \\
iPTH $(\mathrm{pg} / \mathrm{ml})$ & $281.5,399.1$ \\
Albumin $(\mathrm{g} / \mathrm{dL})$ & $3.66 \pm 0.39$ \\
ID weight $(\mathrm{Kg})$ & $1.5,0.85$ \\
ESA dose $(\mathrm{IU} / \mathrm{Kg})$ & $105.8,583.36$ \\
\hline
\end{tabular}

HD: Hemodialysis; URR: Urea reduction ratio; Hb: Hemoglobin; iPTH: intact parathyroid hormone; ID: Interdialytic; ESA: Erythropoietin stimulating agent; IQR: Interquartile range.

Table II demonstrates the indexes related to hemodialysis therapy and Table III compares them among the three groups. Recommended values are in accordance with KDOQI and EBPG guidelines. The dialysis therapy delivered was adequate in terms of urea reduction ratio which was $73.5 \%$. The mean hemoglobin level was $10.98 \pm 1.4 \mathrm{~g} / \mathrm{dL}$, and mean ESA dose given to maintain this level was $105 \mathrm{lU} / \mathrm{kg}$ dry weight. Mean phosphate and calcium levels were $4.75 \pm 1.34 \mathrm{mg} / \mathrm{dL}$ and 9.62 $\pm 0.64 \mathrm{mg} / \mathrm{dL}$, respectively; whereas iPTH was 281.5 $\mathrm{pg} / \mathrm{ml}$. Serum albumin was $3.66 \pm 0.39 \mathrm{mg} / \mathrm{dL}$. Mean interdialytic weight gain (IDWG) was observed to be 1.5 $\mathrm{Kg}$. IDWG was observed to be $1.79 \mathrm{Kg}$ in $10-15$ years group as compared to $1.3 \mathrm{Kg}$ in more than 20 years group. However, no significant difference was observed when compared between the groups. All the indices were comparable to the recommended values. 
Table III: Indexes associated with hemodialysis therapy

\begin{tabular}{|c|c|c|c|c|c|}
\hline \multirow[t]{2}{*}{ Indexes related to hemodialysis* } & \multicolumn{3}{|c|}{ Duration of hemodialysis in years (No. of patients) } & \multirow[t]{2}{*}{ p-value } & \multirow[t]{2}{*}{ Recommended values $†$} \\
\hline & $10-<15(20)$ & $15-20(11)$ & $>20(7)$ & & \\
\hline URR \% & $72.4 \pm 8.7$ & $75.26 \pm 5.8$ & $71.6 \pm 13.1$ & 0.67 & $65-70$ \\
\hline \multicolumn{6}{|l|}{ Anemia } \\
\hline $\mathrm{Hb}(\mathrm{g} / \mathrm{dL})$ & $10.8 \pm 1.35$ & $11.3 \pm 1.66$ & $10.97 \pm 1.96$ & 0.65 & $10-11.5$ \\
\hline ESA dose (IU/kg) & $151.5,149.1$ & $174.4,275$ & $48.7,52$ & 0.21 & \\
\hline \multicolumn{6}{|l|}{ Bone Mineral Metabolism } \\
\hline Phosphate (mg/dL) & $4.7 \pm 1.56$ & $5.1 \pm 0.85$ & $4.4 \pm 1.3$ & 0.55 & $3.5-5.5$ \\
\hline Calcium (mg/dL) & $9.57 \pm 0.68$ & $9.75 \pm 0.6$ & $9.57 \pm 0.63$ & 0.74 & $8.5-9.5$ \\
\hline iPTH $(p g / m l)$ & $321.2,402$ & $269,601.9$ & $324,185.9$ & 0.76 & 2 to 9 times normal \\
\hline \multicolumn{6}{|l|}{ Nutrition } \\
\hline Albumin (g/dL) & $3.7 \pm 0.45$ & $3.67 \pm 0.25$ & $3.4 \pm 0.31$ & 0.18 & $>3.5$ \\
\hline Interdialytic weight gain (kg) & $1.7,0.5$ & $1.5,1$ & $1.5,0.5$ & 0.08 & 1.5 \\
\hline
\end{tabular}

URR: Urea reduction ratio; $\mathrm{Hb}$ : Hemoglobin; ESA: Erythropoietin stimulating agent; iPTH: intact Parathyroid hormone.

TRecommendation values were followed by hemodialysis guidelines of the Kidney Disease Outcomes Quality Initiative (K/DOQI) and the European Best Practices Guidelines for Hemodialysis (EBPG). ${ }^{*}$ Mean + SD, Median, IQR (interquartile range).

\section{DISCUSSION}

Hemodialysis is widely used renal replacement therapy for ESRD. The common perception is that hemodialysis bears poor prognosis; hence, long-term survival on hemodialysis has received little scientific interest. Limited reports are available that describe factors improving survival on hemodialysis. Kurkus et al. reported two patients who had been dialysed for 35 years, briefly interrupted by period of transplant. ${ }^{6}$ Few other series describe patients on chronic hemodialysis, but for a shorter period.7,8 Improvement in dialysis technique and compliance with several guidelines formulated for refining hemodialysis outcome has resulted in better survival. ${ }^{3-5}$ In the absence of registries, regional data on long term survivors of hemodialysis is not available in Pakistan. ${ }^{9}$

The study place is a tertiary-level renal care centre offering regular hemodialysis to 385 patients. Out of these, 38 patients have been on dialysis for more than 10 years. In present study, the mean age at initiation of dialysis was younger in patients on longer hemodialysis duration. This observation was similar to that documented by Otsubo et al. as well as Heaf and colleagues. ${ }^{7,10}$ In this study, eGFR was not calculated at the initiation of dialysis; hence, it cannot be concluded whether aforementioned observation has any significant correlation with recommendation from landmark study named The Initiating Dialysis Early and Late (IDEAL). The IDEAL trial demonstrated that there is no difference in survival and clinical outcomes in CKD stage $V$ patients who had early dialysis initiation and those that were electively delayed. ${ }^{11}$

The primary cause of ESRD in Pakistan is chronic glomerulonephritis and diabetic nephropathy. ${ }^{12}$ Chugh from India documented chronic glomerulonephritis $(37 \%)$ as a primary cause of ESRD in their population, followed by diabetic nephropathy $(14 \%)$, chronic tubulointerstitial nephritis (14\%), and nephrosclerosis (13\%). ${ }^{9}$ Agarwal et al. reported chronic glomerulonephritis as the major cause of renal failure $(49.4 \%)$, trailed by diabetic nephropathy (28.4\%). ${ }^{9}$ Naicker also reported similar etiology of renal failure in South Africa. ${ }^{13}$ Conversely, there is an increasing incidence of ESRD associated with diabetes in Pakistan observed in the last decade and diabetes has become a leading cause with glomerulonephritis to follow. 9 This is similar to epidemiology of ESRD in United States. ${ }^{14}$ In this study, the cause of ESRD mostly remained unknown (47.4\%) either due to small size kidneys or presence of multiple comorbid at presentation. Chronic glomerulo-nephritis is the second most common cause $(15.8 \%)$. An interesting observation is that despite being the leading cause of ESRD, there are only a small number of long term survivors on hemodialysis with ESRD secondary to diabetes $(5.3 \%)$. There were no diabetics in the two groups on more than 15 years of hemodialysis. This was also noted by Otsubo et al. and Heaf et al.7,10 This probably suggests that renal failure secondary to diabetes has poorer prognosis compared to other causes of ESRD.

Survival of ESRD patients is dependent on delivery of adequate hemodialysis. Inadequate hemodialysis is independently associated with increased morbidity and mortality of the patients. ${ }^{3}$ Dialysis Outcomes and Practice Patterns Study (DOPPS) and KDOQI recommend a minimal dialysis dose of single pool KT/V 1.2 and urea reduction ratio (URR) of 65\%.15,16 Owen et al. reported increased mortality associated with URR $<60 \% .{ }^{17}$ In this study, median URR was $73.5 \%$ (IQR 10.95). Similar URR was observed in all the groups. This signifies that the study participants were undergoing adequate dialysis as per guideline recommendation. Recent argument is that URR does not take into account convective urea clearance during ultrafiltration as well as that obtained by residual renal function, and should phase out as a measure of dialysis dose in favour of

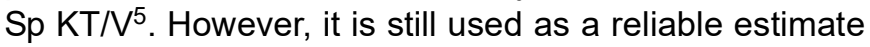
of dose adequacy. 
Adequate patient care is as important as adequate dialysis dose in improving quality of life and survival in hemodialysis patients. ${ }^{5}$ Dialysis, dependent population requires appropriate management of several factors including anemia, mineral bone disease, and nutrition in order to reduce morbidity and mortality. KDOQI recommends maintenance of hemoglobin levels at $10-11.5 \mathrm{~g} / \mathrm{dL}$ in all hemodialysis patients and use of erythropoietin stimulating agents to achieve this level in iron efficient population. ${ }^{18}$ Inverse association of hemoglobin and poor survival have been reported in several studies.19,20 Participants in this study had a mean hemoglobin level of $10.98 \pm 1.4 \mathrm{~g} / \mathrm{dL}$ and average ESA dose prescribed was $105.8 \mathrm{IU}$ per $\mathrm{kg}$ dry weight.

Alteration in mineral metabolism is commonly noted in ESRD patients resulting in soft tissue and vascular calcifications. This may result in vaso-occlusion in coronary, peripheral and cerebral circulation, and eventually resulting in increased morbidity and mortality as reported by Geoffrey et al. and Young et al.21,22 KDOQI guidelines recommend maintenance of corrected serum calcium levels at $8.5-9.5 \mathrm{mg} / \mathrm{dL}$, serum phosphorus $3.5-5.5 \mathrm{mg} / \mathrm{dL}$, and intact parathormone level 2 to 9 times normal.23 These patients had mean serum calcium $9.62 \pm 0.64 \mathrm{mg} / \mathrm{dL}$, serum phosphorus $4.75 \pm 1.34 \mathrm{mg} / \mathrm{dL}$, and $\mathrm{iPTH} 281.5 \mathrm{mg} / \mathrm{dL}$, all within the recommended range.

Good nutritional status is required for surviving chronic hemodialysis. Anorexia secondary to uremia, medications, depression, economic limitation and IL-1, and tumor necrosis factor mediated catabolic stress triggered by hemodialysis itself contribute to malnourishment. Owen et al. and Dwyer et al. documented increased mortality associated with hypoalbuminemia.17,24 Another study showed 6.7 times greater probability of death in presence of serum albumin $<3$ $\mathrm{g} / \mathrm{dL}{ }^{3}$ In the present study, patients had mean albumin $3.66 \pm 0.39 \mathrm{mg} / \mathrm{dL}$ representing satisfactory nutritive status of these patients.

The average interdialytic weight gain (IDWG) was $1.5 \mathrm{Kg}$ which was $<5.7 \%$ in all the patients. This was in accordance with recommended IDWG by DOPPS and KDOQI.15,16 Greater interdialytic weight gain is independent risk factor of CV events, particularly hospitalisation for heart failure / volume overload and mortality. 25

The URR, serum calcium, phosphate, iPTH, albumin and hemoglobin and interdialytic weight gain did not differ between the three different durations of hemodialysis groups.

Complications of chronic hemodialysis such as hepatitis $\mathrm{C}$ infection, carpal tunnel syndrome, spondyloarthro-pathy, paresthesias, acquired cystic kidney disease, parathyroid adenoma, and renal cell carcinoma were also observed with variable frequency among the study participants.
There are few limitations in this study. Young age at initiation of hemodialysis was observed; however, eGFR at which hemodialysis was initiated was not determined. Hence, a lead time bias cannot be confidently excluded. Hemodialysis adequacy was not determined by SpKT/V, which is more accurate means of determining dialysis dose. Quality of life was not evaluated, which is the primary goal of hemodialysis therapy.

To date, this is the first study that presents clinical profile of patients undergoing chronic hemodialysis in Pakistan and highlights the importance of complying with standard guidelines for management of hemodialysis patients and its favourable impact on long term survival on chronic hemodialysis.

\section{CONCLUSION}

Clinical profile and hemodialysis therapy related indices of all the chronic hemodialysis patients satisfied the recommended K/DOQI and EPBG guidelines. By improving quality of dialysis and patient care through compliance with standard guidelines, survival of patients on hemodialysis can be improved in the population similar to developed countries.

Acknowledgement: The present study was carried out for the fulfillment of Fellowship qualification following CPSP approval.

\section{REFERENCES}

1. Jafar TH. The growing burden of chronic kidney disease in Pakistan. N Engl J Med 2006; 354:995-7.

2. Naqvi SJ. Renal diseases in Pakistan - 'Time to act'. J Nephrol Renal Transplant 2009; 2:133-5.

3. Chandrashekar A, Ramakrishnan S, Rangarajan D. Survival analysis of patients on maintenance hemodialysis. Indian $J$ Nephrol 2014; 24:206-13.

4. Tattersall J, Martin-Malo A, Pedrini L, Basci A, Canaud B, Fouque D, et al. EBPG guidelines on dialysis strategies. Nephrology Dialysis Transplantation 2007; 22:ii5-ii21

5. National Kidney Foundation. KDOQI clinical practice guidelines for hemodialysis adequacy: 2015 update. Am J Kidney Dis 2015; 66:884-930

6. Kurkus J, Nykvist M, Lindergard B, Segelmark M. Thirty-five years of hemodialysis: Two case reports as a tribute to Nils Alwall. Am J Kidney Dis 2007; 49:471-6.

7. Otsubo S, Otsubo K, Sugimoto H, Ueda S, Otsubo Y, Otsubo O, et al. Characteristics of patients on hemodialysis therapy for more than 30 years. Ther Apher Dial 2007; 11:274-9.

8. Piccoli GB, Mezza E, Anania P, ladarola AM, Vischi M, Torazza MC, et al. Patients on renal replacement therapy for 20 or more years: A clinical profile. Nephrol Dial Transplant 2002; 17: 1440-9.

9. Ullah K, Butt G, Masroor I, Kanwal K, Kifayat F. Epidemiology of chronic kidney disease in a Pakistani population. Saudi $J$ Kidney Dis Transpl 2015; 26:1307-10

10. Heaf J, Nielsen AH, Hansen HP. Long-term haemodialysis survival. Clin Kidney J 2012; 5:168-9. 
11. Cooper BA, Branley P, Bulfone L, Collins JF, Craig JC, Fraenkel MB, et al. A randomized, controlled trial of early versus late initiation of dialysis. N Engl J Med 2010; 363:609-19.

12. Rizvi SA, Anwar Naqvi SA. Renal replacement therapy in Pakistan. Saudi J Kidney Dis Transpl 1996; 7:404-8.

13. Naicker S. Nephrology in South Africa. Nephrol Dial Transplant 1996; 11:30-1.

14. Saran R, Li Y, Robinson B, Ayanian J, Balkrishnan R, BraggGresham J, et al. US renal data system 2014 annual data report: epidemiology of kidney disease in the United States. Am J Kidney Dis 2015; 66(Suppl 1):Svii, S1-305.

15. Gilmore J. KDOQI clinical practice guidelines and clinical practice recommendations-2006 updates. Nephrol Nurs $J$ 2006; 33:487-8.

16. Pisoni RL, Gillespie BW, Dickinson DM, Chen K, Kutner MH, Wolfe RA. The dialysis outcomes and practice patterns study (DOPPS): Design, data elements, and methodology. Am J Kidney Dis 2004; 44(5 Suppl 2):7-15.

17. Owen WF Jr, Lew NL, Liu Y, Lowrie EG, Lazarus JM. The urea reduction ratio and serum albumin concentration as predictors of mortality in patients undergoing hemodialysis. N Engl J Med 1993; 329:1001-6.

18. Jumaa A. Kidney disease: Improving global outcomes (KDIGO) anemia work group. KDIGO clinical practice guidelines for anemia in chronic kidney disease. Kidney Int 2012; 2: 279-335.
19. Collins AJ. Influence of target hemoglobin in dialysis patients on morbidity and mortality. Kidney Int Suppl 2002; 80:44-8.

20. Avram MM, Blaustein D, Fein PA, Goel N, Chattopadhyay J, Mittman N. Hemoglobin predicts long-term survival in dialysis patients: A 15-year single-center longitudinal study and a correlation trend between prealbumin and hemoglobin. Kidney Int Suppl 2003; 87:S6-11.

21. Block GA, Klassen PS, Lazarus JM, Ofsthun N, Lowrie EG, Chertow GM. Mineral metabolism, mortality, and morbidity in maintenance hemodialysis. J Am Soc Nephrol 2004; 15:2208-18.

22. Young EW, Albert JM, Satayathum S, Goodkin DA, Pisoni RL, Akiba T, et al. Predictors and consequences of altered mineral metabolism: The dialysis outcomes and practice patterns study. Kidney Int 2005; 67:1179-87.

23. Group KDIGOC-MUW. KDIGO 2017 clinical practice guidelines update for the diagnosis, evaluation, prevention, and treatment of chronic kidney disease-mineral and bone disorder (CKDMBD). Kidney Int Suppl 2017; 7:1-59.

24. Dwyer JT, Larive B, Leung J, Rocco MV, Greene T, Burrowes J, et al. Are nutritional status indicators associated with mortality in the hemodialysis (HEMO) study? Kidney Int 2005; 68:1766-76.

25. Cabrera C, Brunelli SM, Rosenbaum D, Anum E, Ramakrishnan K, Jensen $\mathrm{DE}$, et al. A retrospective, longitudinal study estimating the association between interdialytic weight gain and cardiovascular events and death in hemodialysis patients. BMC Nephrol 2015; 16:113. 